Káñina, Rev. Artes y Letras, Univ. de Costa Rica, XLII (1)2018: 33-49/ISSN: 2215-2636

\title{
SUPERANDO MITOS SOBRE LA COMPRENSIÓN LECTORA EN LA POBLACIÓN ESTUDIANTIL UNIVERSITARIA
}

\author{
Overcoming myths about reading comprehension \\ in the university student population
}

\author{
María Gabriela Regueyra Edelman ${ }^{1}$ \\ Sol Argüello Scriba ${ }^{2}$
}

\begin{abstract}
RESUMEN
Este es un ensayo que sintetiza las reflexiones de dos docentes universitarias preocupadas por las dificultades que enfrentan estudiantes de la educación superior, relacionadas con la comprensión lectora. Comparten sus mitos y la manera en que fueron modificando las premisas iniciales para presentar que la comprensión lectora es parte integral de un concepto mayor de la lectoescritura, la cual, a su vez, debe ser analizada desde el contexto de un sistema económico social que reproduce la desigualdad social y cultural de clase. Este trabajo es también el resultado del estudio y análisis de una amplia bibliografía para llegar a plantear principalmente, que la población estudiantil que llega a las aulas universitarias enfrenta una nueva cultura en cada materia académica que debe ser enseñada por la población docente, la cual, a su vez, debe comprender que enseñar en la educación superior implica enseñar a leer, comprender y producir el conocimiento sobre el objeto de estudio disciplinar.

Palabras clave: comprensión lectora, lectoescritura, población estudiantil, universidad, mito.
\end{abstract}

\begin{abstract}
This is an essay that summarizes the thoughts of two university professors concerned about the difficulties faced by students in higher education, related to reading comprehension. They share their myths and how they were modifying the initial premises to conclude that reading comprehension is an integral part of a broader concept of literacy, which in turn must be analyzed from the context of a socio-economic system that reproduces the social and cultural class inequality. This work is also a result of the study and analysis of a wide literature that shows that the student population reaches university classrooms facing a new culture in each academic subject that should be taught by the teaching staff, who, in turn, must understand that teaching in higher education involves teaching to read, understand and produce knowledge about the subject matter.
\end{abstract}

Key Words: reading comprehension, writing skills, student population, university, myth.

${ }^{1}$ Universidad de Costa Rica. M.Sc., profesora e investigadora de la Escuela de Trabajo Social. Costa Rica. Correo electrónico: gabriela.regueyra@ucr.ac.cr

${ }^{2}$ Universidad de Costa Rica. M.L., profesora e investigadora de la Escuela de Filología, Lingüística y Literatura. Costa Rica. Correo electrónico: sol.arguello@ucr.ac.cr

Recepción:28-10-2016 Aceptación:27-01-2017 
Káñina, Rev. Artes y Letras, Univ. de Costa Rica, XLII (1)2018: 33-49/ISSN: 2215-2636

\begin{abstract}
Al inicio
Con más de 25 años de ser docentes universitarias, tomamos conciencia de que en los últimos años, de manera insistente, nos quejamos de que la población estudiantil no realiza las lecturas asignadas, no comprende lo que lee y presenta dificultades para escribir, expresar ideas y establecer un hilo conductor de lo leído. Fueron muchas las oportunidades en que compartimos esta preocupación con otras personas docentes; en esos encuentros y desencuentros decidimos, una docente de la carrera de Trabajo Social junto con una docente de la Escuela de Filología, Lingüística y Literatura, asumir el reto de comprender esta situación y, desde nuestra condición de limitaciones.
\end{abstract}

\title{
Problema que nos convoca
}

La población docente universitaria espera que el estudiantado al ingresar a un nivel universitario tenga entre sus habilidades y destrezas, que permitan la comprensión y análisis crítico de textos, habilidades que favorecen el aprendizaje, el avance académico y el éxito en los estudios universitarios.

Sin embargo, la experiencia docente nos ha demostrado que hoy en día la población estudiantil presenta grandes retos, ya que no todos los estudiantes logran comprender el material básico de los cursos impartidos en este nivel superior y mucho menos alcanzar una posición crítica frente al autor, al respecto Teobaldo y Melgar comentan que:

Es necesario profundizar la idea de que el cambio que afrontan los estudiantes en el nivel terciario implica una ruptura epistemológica. Desde la escuela primaria hasta el nivel secundario generalmente se presenta el conocimiento como producción anónima, atemporal y verdadera. Esta concepción debe ser desarmada en el nivel superior para dar paso a una concepción que caracteriza el conocimiento como histórico, autoral, provisorio y conjetural. El alumno debe aprender a inferir, comparar, confrontar, enmarcar teóricamente, argumentar y refutar. (Teobaldo y Melgar, 2009, p. 3)

Las dificultades de comprender las lecturas asignadas son reconocidas también por la misma población estudiantil, tal como se demuestra en los comentarios que realizaron estudiantes que no continuaron en la carrera de Trabajo Social, documentos que son parte de una investigación realizada en 2010 y 2011 (Regueyra, 2013); esta población manifestó cuán difícil es incorporarse a ese nuevo ambiente académico y obtener el nivel adecuado de comprensión del discurso:

En términos generales Trabajo Social I, me pareció muy confuso, el vocabulario era muy elevado", "y es que pasar del cole a la U, es muy diferente y a uno no lo preparan para ese 
tipo de cambios", "yo solo llevé Trabajo Social I y no entendí mucho cuando estuve, todo era muy confuso". (Regueyra, 2013, p. 8)

Esta es una seria dificultad, ya que el estudiantado de Trabajo Social, durante su formación, debe realizar lecturas sobre diversas temáticas que le den una sólida formación teórica, la cual le permita la comprensión de los problemas sociales, objetos de su trabajo profesional. Para ejercer como profesional debe realizar funciones vinculadas no solo con la comprensión de lo que lee, sino también debe realizar procesos de inferencia y crítica tanto de la realidad social como de las fuentes teóricas y documentales que sustentan las políticas sociales y los proyectos que formula y en los que participa.

Esta situación expresa la necesidad de que, durante la formación profesional, se deben fortalecer las capacidades lectoras en la población estudiantil y para ello es necesario identificar cuál es el nivel de las dificultades que se presentan en ese ámbito. Como también se hace necesario establecer los problemas que conllevan estas dificultades para comprender las lecturas y, en consecuencia, una clara expresión escrita y oral.

\section{¿Qué hicimos y cómo lo hicimos?}

Los primeros esfuerzos se orientaron a identificar publicaciones en la Web sobre "la comprensión lectora en la población estudiantil universitaria" que nos permitiera comprender el problema y luego elaborar propuestas para fortalecer esta habilidad en la población y favorecer su avance académico. Para ello revisamos, resumimos, clasificamos y analizamos diversas fuentes documentales, utilizamos herramientas como Zotero, elaboramos cuadros sobre autores, fechas de publicación de documentos, aportes de cada autor al tema de interés, elección de citas, entre otros. Esta estrategia nos permitió organizar el material y los aprendizajes sobre la comprensión lectora y la lectoescritura en la población estudiantil universitaria, y conforme avanzábamos nos dábamos cuenta del poco conocimiento que sobre este tema tenemos los docentes universitarios.

Con la intención de registrar nuestro aprendizaje, cada una elaboró documentos de manera separada, aportando desde cada disciplina al objeto de interés, con el fin de compartirlo en sesiones de reflexión y construcción conjunta para volver a escribir. Además, de lo aprendido sintetizamos este ensayo para aportar a docentes interesados en la temática y que al igual que nosotras no se consideran expertos.

Compartimos lo aprendido tamizando desde nuestra perspectiva lo que otras personas escribieron. Debemos reconocer que esa tarea no fue fácil, muchas veces armamos y desarmamos 
esquemas de contenido, las propuestas que hoy nos parecían viables para sintetizar todo lo aprendido, días después, modificábamos, volvíamos a leer las lecturas para nuevamente proponer y descomponer lo propuesto, en un armar y desarmar. Es importante resaltar que el impulso y la tranquilidad que encontramos en las lecturas de Carlino, quien siempre fue una inspiración, ya que no solo daban cuenta de su conocimiento sobre cómo enseñar la lectoescritura en la universidad, sino de la manera en que ella enfrentaba sus propios retos para escribir y reestructurar lo escrito (Carlino, 2006b).

A partir de las lecturas realizadas nos acercamos al objeto de estudio y, de esta manera, poco a poco se desmoronaron nuestros mitos que evidenciaban nuestro desconocimiento sobre la dimensión de la comprensión lectora en la población universitaria.

\section{Nuestros mitos: ruptura de la ingenuidad inicial}

Nuestro punto de partida fue nuestra hipótesis empírica: la población estudiantil no tiene hábitos de lectura y no logra comprender lo que lee debido a que la educación secundaria no le permitió desarrollar las habilidades y destrezas necesarias en el campo de la comprensión lectora o que la bibliografía que se les da no es la adecuada, la cual poco a poco se fue derrumbando al encontrar, en las fuentes consultadas, diversas maneras para desmitificar esta afirmación.

Reflexionamos al inicio sobre el significado mismo de la palabra leer, la cual, según el diccionario Latín (2008, p. 653), proviene del verbo latino lego, legere, legi, lectum, verbo cuyo significado nos lo da el mismo diccionario (2008, p. 278): escoger, del griego légein escoger, hablar, decir algo de peso. Las palabras españolas derivadas del latín como lectura, lección, lector, lectivo, lectura, legible o leíble, lego y las derivadas como inteligencia o aleccionar, y muchas más, relacionadas con todo lo que se entiende por los procesos de enseñanza-aprendizaje (Coromines, 2010, p. 333). La lectura, por lo tanto, está intrínsecamente vinculada con hablar y escribir conformando el trinomio básico para la comunicación entre los seres humanos: hablar-leer-escribir, el lenguaje mismo para comunicarnos comprender y apropiarnos del mundo cultural al que pertenecemos. Es por ello que tomamos la decisión de dejar, en la reflexión inicial, el concepto de comprensión lectora para incorporar el de lectoescritura, como uno de los componentes del concepto de capital cultural que se requiere en la educación superior, definido por Ezcurra de la siguiente manera:

...en los alumnos, el concepto de capital cultural remite en particular, si bien no solamente, a algunas habilidades cognitivas y hábitos académicos críticos. Entre otros saber estudiar y saber aprender. Un saber que por una parte engloba un conjunto que Pierre Bourdieu (2005) denomina técnicas trabajo intelectual y arte 
de organizar el aprendizaje y que abarca conocimientos como tomar apuntes en clase, preparar exámenes, estudiar mucha bibliografía y comprenderla, utilizar el diccionario, efectuar una búsqueda documental, crear un índice, usar la biblioteca y herramientas informáticas, y leer cuadros estadísticos y gráficos, entre otros. (2011, p. 62)

Reconocemos que la educación es uno de los medios para aprehender, participar y producir la cultura, está mediatizada y determinada por el origen económico de sus educandos. Las nuevas aproximaciones al objeto de estudio nos llevaron a entender que las deficiencias en la lectoescritura de la población estudiantil universitaria es otra de las manifestaciones de un sistema económico social que reproduce la desigualdad social y cultural de clase, que crea una brecha y que reproduce el limitado capital cultural en algunos sectores de la población estudiantil universitaria, lo que vuelve compleja la idea inicial de que es un problema individual.

Por ello, la comprensión del problema exige una intervención distinta de los esfuerzos, que de manera aislada se han ido introduciendo en la Educación Superior si el interés de las universidades es lograr la formación integral y la producción de conocimiento. Para ello, se debe realizar acciones para superar las limitaciones culturales, propias de la extracción social. Es decir, se requiere, como lo indican Carvajal et al.:

...acompañar el estudiante en el alcance de niveles complejos de lectura, puesto que de no lograr estos niveles se hará difícil para ellos el cumplimiento de tareas académicas tales como la elaboración de ensayos, reseñas, resúmenes, la comprensión de enunciados sobre problemas, tanto técnicos como humanísticos y su consecuente resolución, la comprensión de argumentaciones sobre problemas teóricos, la discusión de los propios programas curriculares de sus cursos y la misma planificación de las actividades y estrategias de aprendizaje basados en los contenidos de los programas de las asignaturas. Mucho más se dificulta la correcta expresión de las ideas, la crítica objetiva a las situaciones de aprendizaje y el ordenamiento de las ideas complejas para construir conocimiento. (2010, p. 139)

Los orígenes de las dificultades de la lectoescritura en la población estudiantil exigen a las universidades realizar propuestas que aseguren la equidad como uno de los pilares del derecho a la educación superior, complementando los esfuerzos realizados en la igualdad en el acceso donde se han concentrado la mayoría de estos en las políticas educativas de las últimas décadas. Estos han favorecido el ingreso a la educación superior de sectores sociales que anteriormente no habían logrado participar de esta cultura socialmente producida, pero deben ir acompañados de garantías de logro en la permanencia y graduación, para que no se convierta el ingreso a la educación superior, como lo indica Tinto (citado por Ezcurra 2011), "como una puerta giratoria” donde al mismo tiempo que ingresa esta población es expulsada, de ahí la siguiente afirmación de Ezcurra: 
En ese marco, y en suma, en América Latina la masificación, al igual que los Estados Unidos, conlleva brechas fortísimas en el egreso según el estatus socioeconómico. Brechas de graduación, brechas de clase, una desigualdad social rotunda. Entonces, y más en general, a escala global, la hipótesis es que ese ciclo extraordinario de masificación supone una inclusión, sí pero una inclusión excluyente, según clases y sectores sociales. Es decir, socialmente condicionada. Otra tendencia estructural medular. (2011, p. 26)

Desde los centros de educación superior es urgente realizar discusiones sobre esta temática, haciendo consciente a la población docente del problema, sus orígenes y manifestaciones. Paralelo a ello, se deben hacer propuestas de políticas institucionales generales que legitimen la equidad para lograr el compromiso de la población docente de proponer alternativas que garanticen el aprendizaje de la población estudiantil, aspectos que permitirán superar la visión focalizada que la señala como único responsable, según propone Estienne y Carlino:

Nuestra propuesta implica cambiar la perspectiva del problema y convertirlo en un tema que involucra a las instituciones de enseñanza y a los docentes que formamos parte de la cultura académica, evitando colocar las dificultades sólo en el alumno, en su falta de lectura o de autonomía lectora. (Estienne \& Carlino, 2004, p. 8)

El desarrollo de la lectoescritura en la universidad coloca a los docentes frente a diversas encrucijadas. Algunos autores como Estienne \& Carlino (2004) plantean que los alumnos sí leen, pero lo hacen de manera distinta a la que nosotros los docentes esperamos:

...los alumnos sí leen, pero leen como pueden o como saben hacerlo. Y esto suele ser distinto a cómo los docentes esperamos que lean. Intentaremos mostrar que nuestras expectativas como profesores (no cumplidas por los alumnos) tampoco aparecen claramente manifiestas. Es decir, los docentes esperamos que los alumnos lean de determinada manera pero no enseñamos a hacerlo y entonces nos quejamos pensando que simplemente no leen. (p. 3)

Conforme fuimos avanzando en el proceso, llegamos a compartir la propuesta de que la universidad tiene su propia cultura, la cual debe ser aprehendida por la población estudiantil y que cada disciplina en sí es una cultura específica y diferente de las demás, tiene su propio vocabulario, su manera de generar conocimiento, su forma de dialogar y de cuestionar. De acuerdo con lo anterior, la población estudiantil se incorpora a esa nueva cultura y esa es tarea de los y las docentes en la educación superior y no de la educación secundaria, puesto que son ámbitos distintos.

La responsabilidad de las universidades de asumir el reto de enseñar a escribir a la población estudiantil universitaria tiene sus orígenes en los países anglosajones. Al respecto, Carlino (2003) comparte en una de sus múltiples investigaciones que las universidades de Canadá 
y Estados Unidos que ella investigó “...han desarrollado una conciencia clara acerca de la centralidad de la escritura como herramienta para aprender cualquier materia y sobre la responsabilidad del nivel superior de promover y enseñar los procesos y las convenciones redaccionales empleados en cada campo disciplinar" (p. 158). Estos países proponen desarrollar procesos de alfabetización académica, preparando a la población estudiantil que ingresa “...en una nueva cultura escrita, para lo cual los estudiantes necesitan participar en las prácticas letradas disciplinares y recibir orientación explícita" (Carlino, 2003, p. 149), para lograr, escribir, sistematizar, elaborar y comunicar conocimiento de su área disciplinar, contraponiendo la idea de que la comprensión lectora se logra de una vez y para siempre y no como un proceso de adquisición de destrezas.

Carlino (2003) plantea que mientras las universidades anglosajonas han iniciado desde hace varias décadas la discusión sobre la alfabetización académica, los países iberoamericanos tenemos pendiente realizar reflexiones sobre la manera en que se enseña la lectoescritura en nuestras universidades, al respecto en sus propias palabras:

...las universidades iberoamericanas tienen pendiente una reflexión profunda acerca del valor que atribuyen a la escritura en la formación de los graduados. Revisar nuestras ideas sobre el escribir, advertir que la producción escrita es un instrumento clave para aprender, reconsiderar, desarrollar y reorganizar el conocimiento sobre una materia-y no sólo un medio de registro o transmisión del saber-, llevaría a plantear qué lugar damos a estos procesos en la educación superior. (p. 159)

\section{Comprendemos el origen y conocemos sus manifestaciones, pero ¿qué podemos hacer?}

Para dar respuesta a esta interrogante, se hizo lo siguiente. Revisamos los aportes de los países de origen anglosajón como Australia, Estados Unidos, Canadá (Marlene Scardamalia y Carl Bereiter, 1992), pioneros en el tema (Carlino, 2005). Luego, desde países de habla castellana como España, Argentina, Colombia y México, por citar algunos, identificamos desde nuestra mirada posturas para comprender, analizar y explicar las dificultades que presenta la población estudiantil universitaria con la lectoescritura, desde visiones estructurales que plantean las limitaciones culturales de las poblaciones estudiantiles, de acuerdo con su procedencia económica y social hasta posturas que centran el problema en el individuo sus habilidades y destrezas cognitivas sin considerar su origen económico social. Estos materiales que revisamos, y que somos conscientes 
son una pequeña muestra de la abundante producción, la clasificamos en las siguientes tendencias, de acuerdo a los factores que la generan:

La lectoescritura como una manifestación de la estructura económica social desigual que genera desventajas culturales en determinados grupos poblaciones (Ezcurra, 2011). La lectoescritura como una manera de apropiación de la cultura académica de cada carrera universitaria (Carlino, 2003; Estienne y Carlino, 2004; Ezcurra, 2011; Carlino, 2013). La lectoescritura como un problema cognitivo (Scardamalia y Bereiter, 1992; Carlino, 2006 ; Braslavsky, 2005; Castro y Sánchez, 2013).

La lectoescritura como un problema técnico instrumental: gramática, composición ortografía, en cursos para toda la comunidad estudiantil, lo mismo que en tesis en la Universidad de Costa Rica (Tapia, 1988).

Desde estas posturas encontramos diversos estudios sobre:

Los hábitos lectores, las habilidades, destrezas y opiniones en relación con la comprensión lectora de la población estudiantil universitaria. (Scardamalia y Bereiter, 1992; Guzmán y García, 2014; Carvajal, Trejos y Montes, 2010)

La relación entre las habilidades y destrezas en la lectoescritura y el impacto en el rendimiento académico y la permanencia en la universidad. (Carvajal, Trejos y Montes, 2010; Dubois, 1995; Ezcurra, 2011; University of Leicester, 2009)

Desde los diferentes acercamientos a la comprensión del problema, encontramos múltiples propuestas para trabajar la lectoescritura en la universidad: Martínez, 2002; Estienne y Carlino, 2004; Carlino, 2004; Carlino, 2005; Congos, 2007; Atehortúa, 2010; Uribe y Camargo, 2011; Alzate, Gómez y Arbeládes, 2012; Carlino, 2013; Castro y Sánchez, 2013, y las ponencias presentadas en las Jornadas sobre "La Lectura y la Escritura en la Formación Académica Docente y Profesional", organizadas por la Universidad Tecnológica Nacional y la Universidad General Sarmientos, realizadas en Argentina en el 2011 (ver anexo 1), entre muchos otros. Sin embargo, para Carlino (2013) las propuestas actuales se pueden agrupar en dos grandes tendencias: "enseñanza en contexto" o "aprestamiento de habilidades o competencias fragmentarias"; y plantea que el debate actual no es instrumental, sino que hay discusiones de fondo; en sus propias palabras lo sintetiza así:

es posible hipotetizar que lo que actualmente está en disputa no será objeto de consenso por bastante tiempo, ya que la polémica afecta hondamente nuestras concepciones de qué es aprender y qué es enseñar, además de involucrar nuestras ideas sobre qué es leer y escribir. (p. 367)

Es un proceso difícil el lograr que las universidades y la población docente universitaria comprendan la responsabilidad que tienen en los procesos de lectoescritura de la población y que las acciones deben superar los cursos instrumentales de redacción y ortografía, incorporando esfuerzos con la participación de la población docente y de diversas disciplinas, como lo plantean Castro y Sánchez (2013): 
Llevó mucho tiempo reconocer que las prácticas letradas académicas están directamente relacionadas con los estilos cognitivos que caracterizan a cada área del saber, y que se necesitan más que cursos de ortografía para acercar a los estudiantes universitarios a los requerimientos y exigencias de la interacción comunicativa especializada". (p. 484)

Para lograr este propósito de la alfabetización académica, será necesario incorporar la lectoescritura en todas las materias de una carrera y superar las propuestas de los cursos específicos de redacción y ortografía aislados de los contenidos y vocabulario propios de la carrera y de la asignatura en particular. Los países de Norteamérica plantean dos propuestas para incorporar la lectoescritura a través del curriculum que se detallan en la siguiente cita:

a) escribir para aprender los contenidos de esa asignatura y b) escribir para apropiarse de las convenciones discursivas de la misma. La vertiente a) parte de la premisa de que los estudiantes sólo asimilan lo enseñado en la medida en que se involucran activamente en los temas de cada materia; por tanto, escribir sobre estos temas es una forma de hacer propio el contenido de lo que se estudia -ya que elaborar un texto exige una máxima actividad-. La vertiente b) señala que escribir permite internalizar los patrones comunicacionales específicos de cada disciplina, y que aprender en la universidad es irse incorporando a la cultura escrita de un campo de estudios, motivo por el cual es preciso que los profesores se ocupen no solo de transmitir sus conceptos sino de enseñar su prácticas lectoras y escritoras. (Carlino, 2006, p. 151)

Para el logro de la alfabetización académica, se debe establecer cambios en la manera en que el docente asume su papel, en palabras de Dubois (1995):

nos dimos cuenta de que debíamos enfrentar nuestra práctica docente de una manera distinta, tratando de crear en el salón de clases un ambiente en el que las relaciones humanas tuvieran tanta importancia como el aprendizaje de conocimientos y en el que la lectura y la escritura no fueran hechas para nosotros los profesores, sino para que el estudiante descubriera su propio potencial como lector y escritor. Es lo que hemos intentado hacer." (p. 8)

Por su parte, Estienne y Carlino (2004) sugieren también que los docentes debemos asumir un papel más comprometido en el aprendizaje de la población estudiantil, indicando que la queja docente debe ceder paso a la enseñanza: en este caso, a la enseñanza de una práctica lectora. Y enseñar prácticas no es lo mismo que enseñar conceptos. (Estienne \& Carlino, 2004, p. 3). Por ello, 
el proceso de incorporación de la comprensión lectora y la habilidad de escribir en los estudiantes universitarios es una tarea que involucra todas las instancias universitarias, debe constituirse parte del quehacer académico inclusivo y con miras a ofrecer una universidad comprometida con la sociedad.

De esta manera, el proceso de investigación iniciado nos fue conduciendo por diversos caminos donde encontramos nuevos retos distintos a las primeras interrogantes que nos convocaron; y comprendimos que las manifestaciones y los abordajes de la lectoescritura en la universidad conforman un reto mucho más complejo que apenas estamos comenzando a comprender. Por ello, la tarea aún está pendiente, y entre los retos está continuar en la búsqueda de investigaciones y discusiones que se han dado en Costa Rica, así como las posibles maneras de trabajar desde nuestra realidad universitaria costarricense el tema de la lectoescritura en la universidad.

\section{Bibliografía}

Álzate, María; Gómez, Miguel y Arbeláez, Martha. (2012). Enseñar en la Universidad Saberes, Prácticas y Textualidad. Revista Visión Electrónica, 1, 137-140. Recuperado de http://revistas.udistrital.edu.co/ojs/index.php/visele/article/viewFile/3756/5539. Consulta: 2014-2015.

Atehortua, Jaime. (2010). Mediador de Lecto-Escritura y Técnicas de Estudio. (PDF) Recuperado de

http://www.uco.edu.co/academica/pedagogos/Documents/Libro\%20Mediador\%20de\%20L ectoescritura\%20y\%20Técnicas\%20de\%20Estudio.pdf. Consulta: 2014-2015.

Braslavsky, Berta. (2005). Enseñar a Entender lo que se lee: La Alfabetización en la familia y en la Escuela. Recuperado de http://www.eduteca.mex.tl/imagesnew/8/8/4/5/0/Alfabetización.pdf. Consulta: 2014-2015.

Carlino, Paula. (2003). Alfabetización académica: un cambio necesario, algunas alternativas posibles. Revista EDUCERE, Investigación, 20, 409-420. Recuperado de http://portal.fagro.edu.uy/docs/uensenia/Carlino_Alfabetización\%20académica.pdf. Consulta: 2014-2015.

Carlino, Paula. (2004). ¿De qué modos incentivar en nuestras instituciones la responsabilidad compartida por cómo se lee y se escribe en la Universidad IFD. I Congreso Internacional Educación, Lenguaje y Sociedad Tensiones Educativas en América Latina. Organizado pro el Instituto para el Estudio de la Educación, el Lenguaje y la Sociedad, Facultad de Ciencias Humanas, Universidad Nacional de la Pampa. Recuperado de http://www.aacademica.org/paula.carlino/86.pdf. Consulta: 2014-2015. 
Káñina, Rev. Artes y Letras, Univ. de Costa Rica, XLII (1)2018: 33-49/ISSN: 2215-2636

Carlino, Paula. (2005). Representaciones sobre la escritura y formas de enseñar en la Universidad de América del Norte. (PDF). Revista de Educación, 336, 143-168. Recuperado de http://www .revistaeducacion.educacion.es/re336/re336_09.pdf. Consulta: 2014-2015.

Carlino, Paula. $\left(2006^{\mathrm{a}}\right)$. Escribir, leer y aprender en la universidad Una introducción a la alfabetización académica. Editorial: Fondo de Cultura Económico de Argentina. Recuperado https://aprendeenlinea.udea.edu.co/revistas/index.php/unip/article/viewFile/12132/11016 Consulta: 2014-2015.

Carlino, Paula. $\left(2006^{\mathrm{b}}\right)$. La escritura en la investigación (PDF). Documentos de trabajo Escuela de Educación Universidad de San Andrés, 19. Recuperado de http://live.v1.udesa.edu.ar/files/ESCEDU/DT/DT19-CARLINO.pdf Consulta: 2014-2015.

Carlino, Paula. (2013). Alfabetización Académica Diez años Después. (PDF). Recuperado de http://www.redalyc.org/pdf/140/14025774003.pdf. Consulta: 2014-2015.

Carvajal, Paula, Trejos, Alvaro, Montes, Héctor. (2010). Competencia Lectora de los Estudiantes de la Universidad Tecnológica De Pereira y Rendimiento Académico. (PDF). Recuperado de http://dialnet.unirioja.es/descarga/articulo/4562469.pdf. Consulta: 2014-2015.

Castro, María y Sánchez, Martín. (2013). La Expresión de Opinión en Textos Académicos Escritos por Estudiantes Universitarios. (PDF). Recuperado de http://www.redalyc.org/pdf/140/140 25774008.pdf Consulta: 2014-2015.

Congos, Dennis. (2007). Identifying Problems that lead to slow Reading. (PDF). Recuperado de http://sarconline.sdes.ucf.edu/files/files/2014/01/Identifying_Problems_That_Lead1.pdf Consulta: 2014-2015.

Coromines, Jean. (2010). Breve diccionario etimológico de la lengua castellana. España: Editorial Gredos.

Dubois, María. (1995). Lectura Escritura y Formación Docente. (PDF). Recuperado de http://www.lecturayvida.fahce.unlp.edu.ar/numeros/a16n2/16_02_Dubois.pdf. Consulta: 2015

Ezcurra, Ana. (2011). Igualdad en Educación Superior: Un desafío Mundial. Buenos Aires, Argentina: Editorial UNGS.

Estienne, Viviana y Carlino, Paula. (2004). Informes de Investigación y Ensayos Inéditos: Leer en la Universidad Enseñar y Aprender una Cultura Nueva. (PDF). Recuperado de https://aprendeenlinea.udea.edu.co/revistas/index.php/unip/article/viewFile/15150/13195 Consulta: 2014-2015.

Diccionario Latín. (2008). Vox Spes. Barcelona: Larousse Editorial, S.L.

Guzmán, Fernando y García, Eduardo. (2014). Los Hábitos lectoescritores en los alumnos Universitarios. (PDF). Revista Eléctronica Interuniversitaria de Formación del Profesorado, 17 (3), 79-92. Recuperado de http://revistas.um.es/reifop/article/view/204071 Consulta: 2014-2015. 
Káñina, Rev. Artes y Letras, Univ. de Costa Rica, XLII (1)2018: 33-49/ISSN: 2215-2636

Martínez, María. (2002). Estrategias de Lectura y Escritura de Textos Perspectivas Teóricas y Talleres. Cali, Colombia. Recuperado de http://www.ceip.edu.uy/IFS/documentos/2015/lengua/bibliografia/martinez_estrategiaslect uraescritura.pdf .Consulta: 2014-2015.

Regueyra, María Gabriela. (2013). Aprendizajes sobre la población estudiantil que no continúa y el derecho a la educación superior. Ponencia presentada en la III Conferencia Latinoamericana sobre el abandono en la Educación Superior. Recuperado de http://www.alfaguia.org/wwwalfa/images/ponencias/clabesIII/LT_1/ponencia_completa_8.pdf Consulta: 2014-2015.

Scardamalia, Marlene y Bereiter, Carl. (1992). Dos Modelos Explicativos de los Procesos de Composición Escrita. Recuperado de http://ciequilmes.com.ar/wpcontent/uploads/2013/03/PLEN_Scardamalia-Bereiter_Unidad_11.pdf. Consulta: 20142015.

Tapia, Jesús. (1988). Dificultades de comprensión lectora: origen, entrenamiento y evaluación. (PDF). Recuperado de http://www.juntadeandalucia.es/averroes/ cepco3/competencias/lengua/aspgenerales/Dific ultades_CL.pdf. Consulta: 2014-2015.

Teobaldo, Marta y Melgar, Sara. (2009). Competencias en la comprensión lectora y producción escrita. Alumnos de Institutos Superiores de Formación Docente: Dificultades de los estudiantes de ler. año. (PDF). Recuperado de http://www.buenosaires.gob.ar/sites/gcaba/file /2009_competencias_en_la_comprension _ lectora_y_produccion_escrita.pdf Consulta: 2014-2015.

Segura Mungía, Santiago. (2013). Nuevo Diccionario Etimológico Latín-Español y de voces derivadas. España: UDeusto.

University of Leicester. (2009). Improving Your Reading Skills. (PDF). Recuperado de https://www2.le.ac.uk/offices/ld/resources/study-guides-pdfs/studyskills\%20pdfs/improving-reading-v0.1.pdf . Consulta: 2014-2015.

Uribe, Graciela y Camargo, Zahyra. (2011). Prácticas de Lectura y Escritura Académicas en la Universidad Colombiana. (PDF). Recuperado de http://revistas.javeriana.edu.co/index.php/MAGIS/article/view/3543. Consulta: 2014-2015. 
Káñina, Rev. Artes y Letras, Univ. de Costa Rica, XLII (1)2018: 33-49/ISSN: 2215-2636

\section{Anexo 1}

Jornadas sobre "La Lectura y la Escritura en la Formación Académica Docente y Profesional", organizadas por la Universidad Tecnológica Nacional y la Universidad General Sarmientos y realizadas en Argentina en el 2011. Consulta: 2014-2015:

Ponencia

Autor

País

Institución

Alfabetizaciones múltiples en la formación docente inicial: conjugando lo digital y lo analógico, lo lingüístico y lo disciplinar, lo coloquial y lo académico.

Cristina Rafaela Ricci

Argentina

Instituto Superior de Formación Docente Buenos Aires.

Análisis de condiciones didácticas en la enseñanza académica. la lectura y la escritura en la formación de profesores

Graciela María Elena Fernández, María Viviana Izuzquiza, María Alejandra Ballester , María Pía

Barrón, María Daniela Eizaguirre , Florencia Zanotti

Argentina

Universidad Nacional del Centro de la Provincia De Buenos Aires

(UNICEN)

Aprender ciencia mediante la escritura

Mg. Graciela Iturrioz

Argentina

Universidad Nacional de la Patagonia San Juan Bosco

Argumentación en biología. el caso de los estudiantes de la licenciatura en ciencias biológicas de la U.N.N.E.

Margarita C. Ortiz, Aníbal R. Bar.

Argentina

Universidad Nacional del Nordeste

Argumentación y pensamiento crítico articulados en la alfabetización académica: reflexiones docentes

Silvina Douglas de Sirgo, Esther López, Constanza Padilla

Argentina

Universidad Nacional de Tucumán

Ayudas pedagógicas y recursos para escribir en los primeros años universitarios.

Facultad de Psicología Universidad de Buenos Aires

Emilse Diment

Argentina

Universidad de Buenos Aires

Bitácoras

Héctor Mauas, Alberto Casasnovas

Argentina

Universidad de Buenos Aires 
Dos experiencias de uso de NTIC en el taller de escritura

Analía Reale

Argentina

Universidad de Buenos Aires

El lenguaje y la matemática

Silvia Pareja, Dalcy flores

Argentina

Universidad Nacional De Salta Argentina

El problema de la lectura y la escritura académica en los estudiantes, ¿y en los docentes?

Adela Castronovo, Alicia Zamudio, Diego Picotto

Argentina

Universidad Nacional de Lanús

El rol de las estrategias cognitivas y metacognitivas como herramientas para fomentar la autonomía del alumno en la lectocomprensión en lengua extranjera

Fabián Negrelli, Daniela Moyetta

Argentina

Universidad Nacional de Córdoba.

Elogio de la situación argumentativa

Nora Isabel Muñoz, Mónica Beatriz Musci

Argentina

Universidad Nacional de la Patagonia Austral

¿Enseñar a leer, es dar la palabra?

Adén Fernando Peluffo

Argentina

Universidad Nacional de Lújan

Escribir para aprender biología: una propuesta de trabajo que integra la escritura con la disciplina en el primer año de la universidad

Patricia Iglesia, Ana de Micheli

Argentina

Universidad de Buenos Aires

La escritura académica en la formación de grado reflexiones a partir de la puesta en práctica de talleres específicos tutoriales.

María Cecilia Rigonat, Claudia García

Argentina

Universidad Atlántida Argentina

La importancia de la lectura y la escritura en las asignaturas prácticas de la carrera de educación física

Walter N. Tosca

Argentina

Universidad Nacional de La Matanza Buenos Aires Argentina 
La innovación, práctica que distingue al docente.

Norma Salvatierra, Fabiana Maceiras, Adriana Maceira

Argentina

Universidad Nacional de Lomas de Zamora. Buenos Aires Argentina

La lectura en el curso de ingreso. intervención didáctica, experiencia piloto en la UNLAM.

Ana Bidiña, Liliana Luppi, Nora Smael

Argentina

Universidad Nacional de la Matanza, Buenos Aires Argentina

La lectura y la escritura en universidades y profesorados. relevamiento de algunos aspectos y reflexión global sobre la problemática.

Antonio Carlos Cámpora

Argentina

Instituto Superior del Profesorado "Dr. Joaquín V. González"

La productividad de la lectura: una experiencia de Formación docente

María del carmen Gurtubay, Estela Kallay y Andrea Vilari

Argentina

Instituto superior de formación docente y técnica \#53 Argentina

La reflexión sobre el nivel morfosintáctico y léxico en la comprensión de textos: valor discursivo de las nominalizaciones.

Patricia Supisiche, María Eugenia Cendoya, Clara Cacciavillani, Ana Julia Cappuccino

Argentina

Universidad Blas Pascal Córdoba

Las demandas de explicaciones en exámenes de la carrera de biología.

Aníbal R. Bar, Sergio David Valenzuela

Argentina

Universidad Nacional del Nordeste

Las prácticas de lectura y escritura académicas desde el aula virtual

Marcela Paula González

Argentina

Universidad Nacional de Mar de Plata

Leer y escribir en el ingreso a la universidad: una integración necesaria.

María Ignacia Dorronzoro

Argentina

Universidad Nacional de Luján, Buenos Aires Argentina

Leer y escribir en la escuela media y la universidad: el malestar docente.

Jorge Warley

Argentina

Universidad de Buenos Aires Universidad Nacional de la Pampa

Leer y escribir en la universidad. Propuesta de intervención para los primeros años.

Viviana M. Estienne

Argentina

Universidad de las Ciencias Empresariales 
Leer, escribir y argumentar una aproximación a las nociones de pensamiento crítico y argumentación desde la perspectiva de docentes primarios tucumanos.

María Elene Molina, María Virginia Hael.

Argentina

Universidad Nacional de Tucumán - CIUNT.

Lenguaje, cognición e iniciación académica. una historia sobre la escritura para reflexionar en tres niveles.

Diana Rabinovich y Guadalupe Reinoso

Argentina

Universidad Nacional de Córdoba, Facultad de Filosofía y Humanidades

Los inicios de la alfabetización universitaria: el lugar de la lectura y la escritura.

Emilia Garmendia, Mariela Senger, Carolina Folgar.

Argentina

Universidad Nacional de Mar del Plata

Artefactos visuales en textos académicos de historia y literatura a nivel de doctorado.

Cristian Aravena-castro

Chile

Pontificia - Universidad Católica de Valparaíso Chile

Alcances, limitaciones y retos en la enseñanza de la escritura en la universidad (dos casos: Colombia y Argentina)

Adriana Cecilia Goyes Morán, Irene Klein.

Colombia

Pontificia Universidad Javeriana-Puj Sede Bogotá

La percepción que tienen estudiantes de postgrado sobre la escritura

Luz Marina Martínez Peña

Colombia

Universidad del Bosque, Bogotá

La Lectura y la Escritura en las Prácticas Docentes Universitarias.

Luceli Patiño Garzó

Colombia

Universidad de Ibagué

Déficit en comprensión lectora en el nivel superior: ¿cuáles son las creencias de los estudiantes? Marisol López Romero

México

Universidad Autónoma de Sinaloa

El uso de la inferencia en la comprensión del discurso histórico.

Brenda Selene de la Cruz Salas, Hugo Bernales Casillas

México

Universidad de Guadalajara 
Káñina, Rev. Artes y Letras, Univ. de Costa Rica, XLII (1)2018: 33-49/ISSN: 2215-2636

Lectura y escritura: factor fundamental en la adquisición de competencias tecnológicas e informativas.

Angélica Barbosa, Marabel Martín de la Cruz Ruvalcaba, México

Universidad de Guadalajara

Los docentes universitarios de español como primera lengua (1l), ¿escriben y publican?

Claudia Albarrán Ampudia, Dafne Gonzáles Solís

México

Instituto Tecnológico Autónomo de México

La escritura académica de los estudiantes de Ingeniería de la universidad nacional de Itapúa Mirtha Dalila Lugo, Paraguay.

Universidad Nacional de Itapúa, Paraguay

Esta obra está bajo una licencia de Creative Commons Reconocimiento-NoComercial-SinObraDerivada 4.0 Internacional. 